\title{
A FRAMEWORK FOR THE FLEXIBLE GROUPING OF PRODUCTS FOR DISASSEMBLY
}

\author{
SHIVAKUMAR VISWANATHAN \\ Sustainable Design Laboratory, Engineering Management Department, \\ University of Missouri-Rolla, Rolla, Missouri-65409, USA \\ DR. VENKAT ALLADA* \\ Sustainable Design Laboratory, 205 Engineering Management Department, \\ University of Missouri-Rolla, Rolla, Missouri-65409, USA \\ E-mail: allada@umr.edu
}

Received 9 September 1998

Accepted 27 September 1999

\begin{abstract}
The increased scrutiny of a product's effects on the environment has led industry to embrace Environment Conscious Design and Manufacture (ECDM) practices. Disassembly of a product at the end of its useful life is an integral part of the ECDM paradigm. Disassembly facilitates the end-of-product life options such as recycling, remanufacture, reuse and safe disposal. As industries take active steps to move to a "greener" economy, the emergence of dedicated disassembly facilities is foreseeable. The increasing variety of new products requires that these disassembly factories be able to handle a wide spectrum of products. Today, dedicated disassembly facilities exist for disassembling high value products such as computers and automobiles. However, the flexibility of a disassembly facility is particularly important to accommodate the disassembly of moderate to low value, and high variety consumer products. We address this issue by proposing a framework for the formation of product groups based on their disassembly characteristics as a step towards increasing the flexibility of disassembly factories. A two-level hierarchy of generic disassembly characteristics has been employed to evaluate the similarity of diverse products. We have used a modified version of the "growing neural gas" neural network model to classify the products. The first level or primary Disassembly Product Groups (DPGs) are obtained based on similarities in product structure. They are further subdivided into secondary DPGs based on the disassembly resource requirements of the products. An implementation for the disassembly-oriented grouping using a few example products has been carried out for demonstration purposes.
\end{abstract}

Keywords: Disassembly factory, product grouping, neural network.

\section{Introduction}

Increasing pressure due to government regulations, public concern and rising costs has influenced the industry to adopt Environment Conscious Design and Manufacturing (ECDM) practices. The overall goal of the ECDM practices is to ultimately yield products whose environmental impact is minimal, through every stage of the product's life. ${ }^{1-3}$ In keeping with this goal of ECDM, it is critical to consider the product's environmental effects at the end of its useful life. Disassembly is an important process affecting the product retirement or end-of-life (EOL). ${ }^{4,5}$ It is through selective and systematic disassembly that a product can be released for further use or safe disposal. As can be seen from Fig. 1, material recycling and part recycling can then be used to reroute the product's resources into the manufacturing system. Part recycling implies the reuse of the parts or subassemblies of the product either "as is", or after remanufacture, for the same

\footnotetext{
*Author to whom correspondence is to be sent.
} 


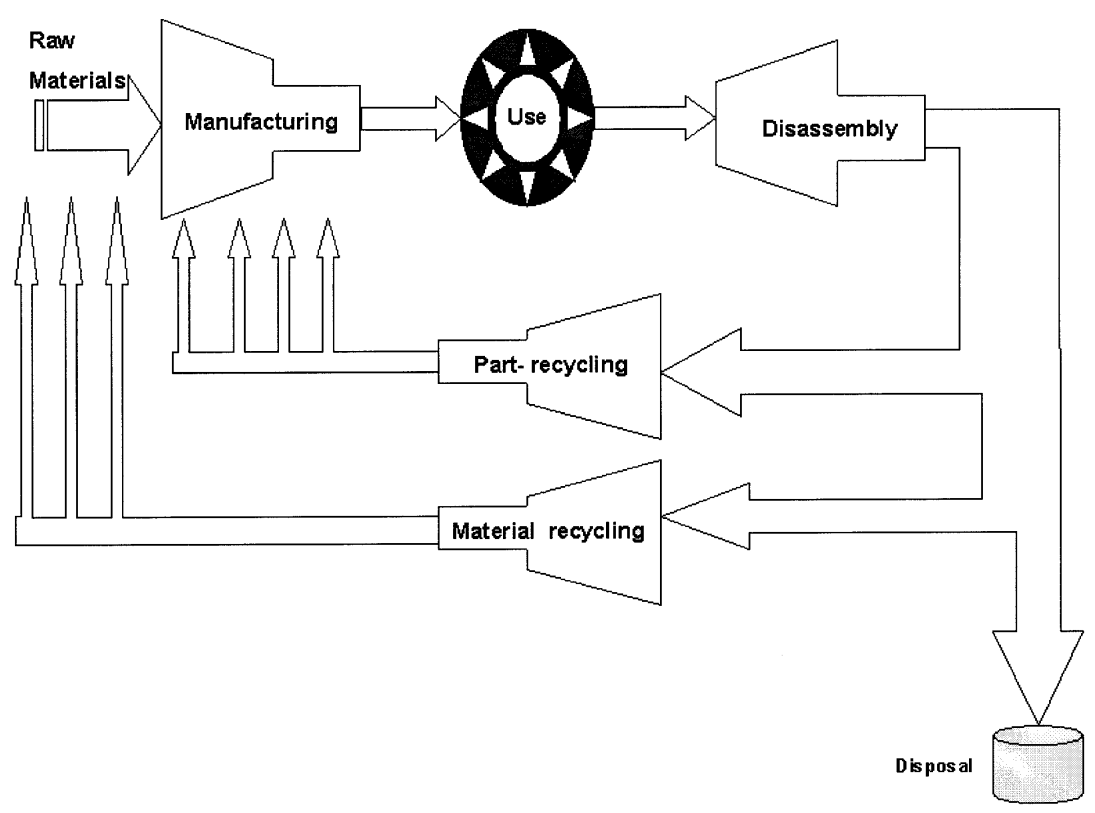

Fig. 1. Product life-cycle.

or a different product. The high cost of disassembly is a major hurdle for the profitable implementation of these ecologically friendly EOL strategies. The economic incubus involved has sparked off active research into different facets of disassembly. Though interdependent, the principal categories into which research work in disassembly can be divided is as follows: ${ }^{6}$

(a) Product design: Product design has a major influence on the disassembly of a product. The main emphasis has been on developing the design guidelines to guide designers in the conceptual and the final design stage. The emphasis has also been on the development of disassembly evaluation methodologies and tools.

(b) Process Design: The thrust of this area is in developing methodologies and software tools to select disassembly strategies and configure disassembly systems.

(c) System Design: This involves the issues affecting the actual performance of the disassembly. It involves the economic justification for the disassembly systems, the configuration of the facility (manual or automated) and the organization of a logistic network for the implementation of the disassembly system.

In this paper, the primary emphasis is on the system design aspect of disassembly. Many researchers have attempted to address the issues involved in disassembly system design. Sarkis ${ }^{7}$ and $\mathrm{Uzsoy}^{8}$ have proposed methodologies addressing the supply chain management aspects affecting environment related processing of products. An optimization methodology for remanufacture oriented systems has been presented by Hoskino et al. ${ }^{9}$ and Stuart et al. ${ }^{10}$ Gupta and Taleb $^{11}$ have presented a scheduling algorithm for a disassembly system.

\subsection{The disassembly factory}

With the increasing volume and variety of products that need to be disassembled, the emergence of dedicated facilities to handle the enormity of this disassembly task in the near future can be foreseen. ${ }^{12,13}$ However, in order to set up these disassembly factories a number of critical issues need to be addressed, including, ${ }^{12-14}$

(a) Wide variety of products and limited or no knowledge of their properties.

(b) Lack of knowledge about the state of products after use.

(c) Lack of knowledge of volume of the products to be disassembled.

(d) Changes in process technology.

(e) Frequent changes in market price/requirement of the salvaged materials and parts.

(f) Changes in legislative requirements. 
(g) Plant location problems.

(h) Resource allocation to carry out disassembly.

A closely related issue to the resource allocation factor for the disassembly factories (factor (h) above), is flexibility, i.e., the ability to handle a wide variety of products in different abuse states. The "flexibility" is based to a large extent on the disassembly tools used ${ }^{15}$ and the types of products that have to be disassembled. The desired "flexibility" could thus be incorporated into the system by grouping different kinds of products based on the similarity of their disassembly characteristics and using a cellular system to carry out the disassembly of each of these groups efficiently. This is the fundamental idea behind the Group Technology (GT) in which part groups (and manufacturing cells) are formed based on the design and manufacturing similarities. ${ }^{16,17}$

Hentschel et al. ${ }^{14}$ proposed the formation of cellular disassembly systems based on the GT concept. In their work, the cell concept was demonstrated for the disassembly of cathode ray tubes (CRTs) from television sets and computer monitors. This grouping was performed using a fuzzy C-means algorithm, based on the design and usage characteristics of the CRTs.
Today, the focus on flexible disassembly is concentrated primarily on high value products like automobiles and computers. ${ }^{15,18}$ However, there exist a large number of products for which setting up dedicated disassembly facilities cannot be economically justified. This is especially true for products from the moderate to low value, high variety, low volume sector, for example, household appliances (such as electric toasters and vacuum cleaners). In order to make the disassembly of these types of products viable, the factories will be required to handle diverse products, thus, making factory flexibility extremely critical.

In the following sections, a framework is proposed for the grouping of the diverse products into Disassembly Product Groups (DPGs) based on the similarity of their disassembly characteristics. The implementation of the framework using a modified form of the "Growing Neural Gas" neural network model $^{19}$ is also described.

\section{Grouping Rationale}

The scope of application for the DPGs is shown in Fig. 2. The approach proposed here is to obtain a "first cut" grouping of the products from the product universe. The product universe consists of all the

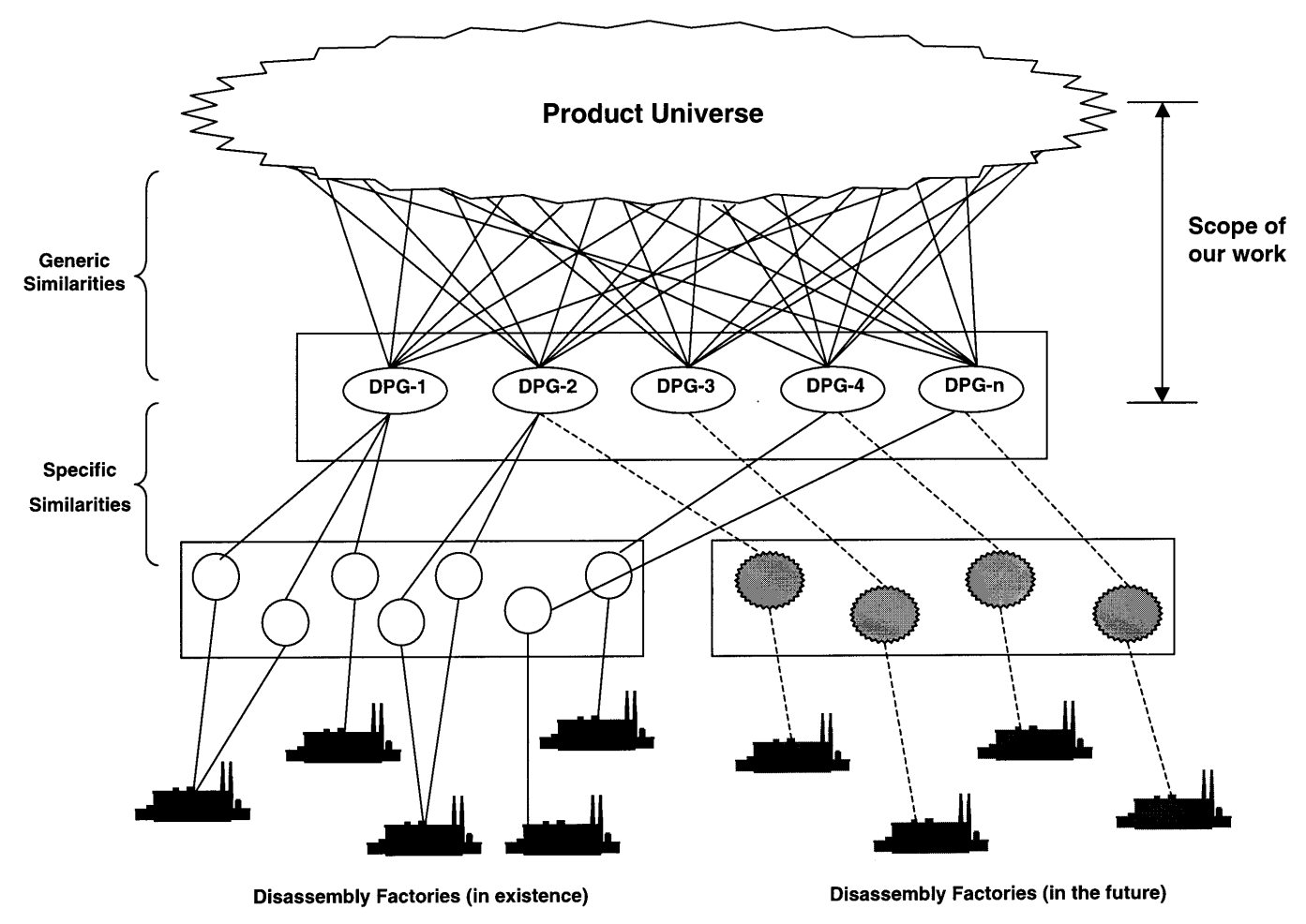

Fig. 2. Product grouping for flexible disassembly factories. 
possible products that require to be disassembled at the end of their useful lives before further processing can be carried out on them. One approach to form groups is the use of a "bottom-up" approach in which a facility's available resources are matched with the disassembly requirements of the different products. This leads to a narrow product grouping constrained by the resources of the factories and other logistic issues that are known a priori. Rather than follow this approach, a "top-down" approach is used to form DPGs, using the product's attributes and generic disassembly characteristics. Thus, the DPGs formed are independent of the constraints of a specific disassembly factory. The DPGs formed could be used for the following purposes:

- Reducing the product search space by forming smaller and more tractable groups that could be further analyzed to match the needs of an existing disassembly facility.

- Planning for new disassembly factories.

It is to be noted that the DPGs represent a high level grouping based on disassembly characteristics that are common to a large variety of products so as to facilitate the formation of groups across product platforms.

\subsection{Terms and definitions}

Before proceeding further, the terms and definitions used in the current study are presented below:

Dividend and Quotients: The entity on which the disassembly operation is being performed is called the "dividend". The entities that are removed from the dividend during the disassembly operation in question are called the "quotients". Refer to Fig. 3.

Atomic moiety: A quotient that requires no further disassembly, according to the disassembly plan, is called an atomic moiety (A-moiety). An A-moiety might thus be a single part, a subassembly or a clump but does not include the fasteners that are removed.

Fastener moieties: The quotients that are fasteners are called, simply, fastener moieties (F-moieties).

Primary Assembly: The product assembly at the end of its useful life is called the Primary Assembly. The term product will be used interchangeably with primary assembly in this paper.

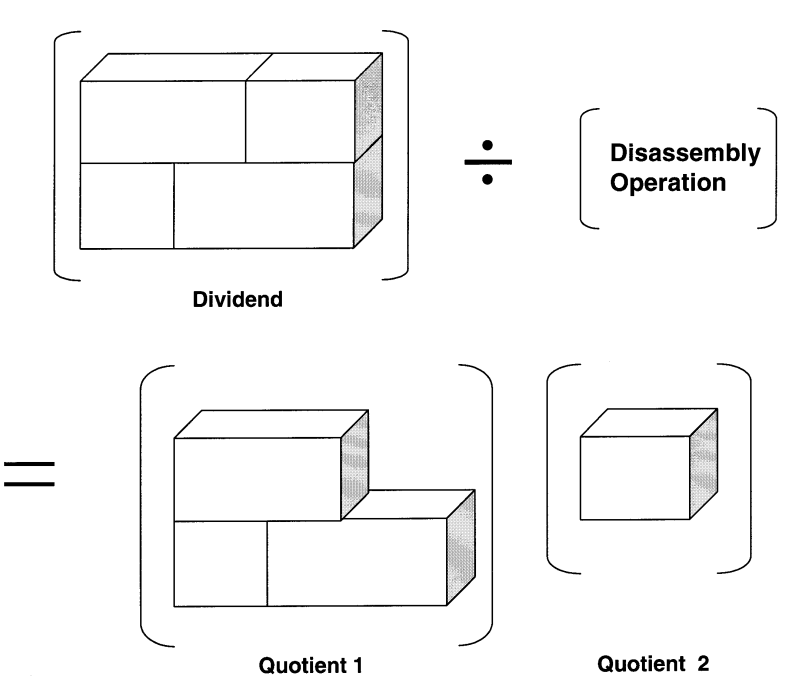

Fig. 3. Relationship between disassembly dividend and quotient.

Starting Assembly (SA): The first dividend to be disassembled may not necessarily be the primary assembly. So, the first dividend is simply called the Starting Assembly.

Disassembly operation (D): A disassembly operation is one that results in the creation of a quotient entity. These include the task of undoing the fasteners and the removal of quotients.

Auxiliary operations ( $\boldsymbol{A} \boldsymbol{D})$ : The auxiliary operations are the ones that are conducted either prior to disassembly (cleaning, inspection, etc.), during disassembly (oiling, inspection, etc.) or immediately after disassembly (sorting, etc.).

The relationship of the above definitions can be seen in Fig. 4 as a schematic of a hypothetical disassembly factory.

\subsection{Disassembly product/process attribute hierarchy}

The principal determinants of disassembly that are relevant to DPG formation are:

1. The Ends: This refers to the materials, parts and subassemblies that are salvaged from the product as a result of the disassembly.

2. The Means: This refers to the tools, fixtures and other resources that are required to perform the disassembly.

3. The Process: This refers to the specific way in which disassembly is carried out and is 


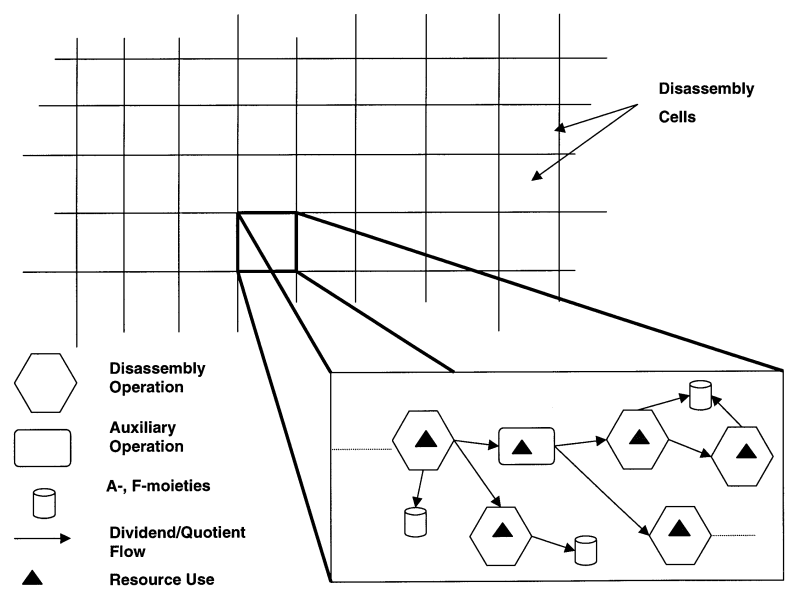

Fig. 4. Schematic of structure of a disassembly factory.

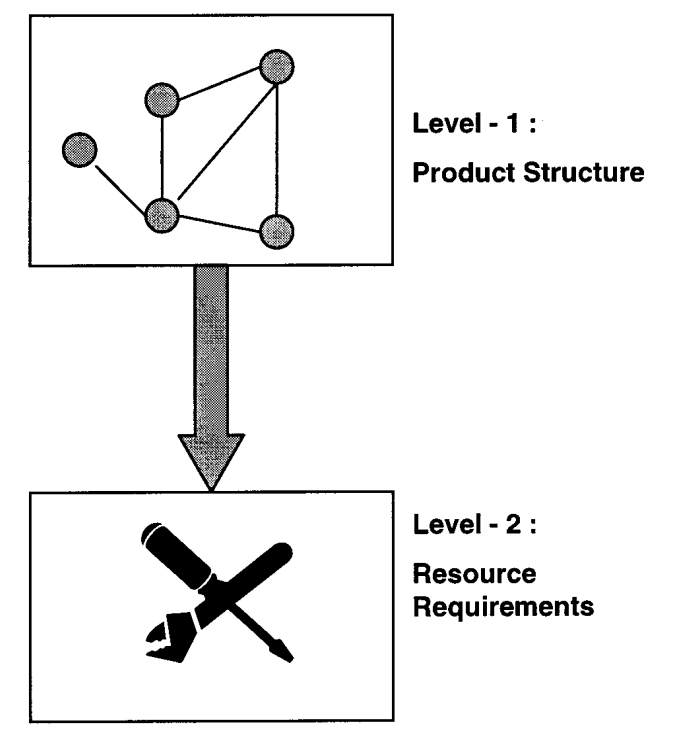

Fig. 5. DPG formation strategy.

dictated by the disassembly sequence. The process determines how and when the means are to be used.

Using these principal determinants stated above, a two-level hierarchy of disassembly attributes was developed for the formation of the DPGs as shown in Fig. 5.

Each attribute is given an ordinal value on a scale from one to ten based on the product structure information and the disassembly sequence. An important assumption is that this information is available a priori and that the disassembly is being carried out according to a predetermined process plan. It is to be noted that due to the diversity of products to be grouped, specific part recovery was not considered for the DPG formation. The attributes used in this two-level hierarchy are described below.

\subsubsection{The product structure}

In this paper, the product structure refers to the physical characteristics of the product such as size, mass and shape. The DPGs formed due to the first level similarity are very general and are called the primary DPGs. The attributes in this level are derived from the geometry of the primary assembly and the quotients formed during disassembly.

(a) Size/mass of the starting assembly.

(b) Average size/mass of the quotients.

(c) Shape regularity of the starting assembly.

(d) Average shape regularity of the quotients.

(a) Size/mass of the starting assembly $\left(\mathrm{M}_{\mathrm{SA}}\right)$ This attribute provides a measure of the handling and space requirements of the starting assembly to be disassembled. Though a volumetrically large starting assembly may not be very heavy and vice versa, size and mass are largely correlated as far as the handling requirements are concerned. The scale ranges from one indicating a very small/light starting assembly and a rating of ten indicates a very large/heavy starting assembly. The user specifies the products defining the limits of the scale. For example, $\mathbf{M}_{\mathbf{S A}}=10$ for a tractor and $\mathbf{M}_{\mathbf{S A}}=3.5$ for a table fan.

\section{(b) Average size/mass of the quotients $\left(M_{q}\right)$}

The size and mass attributes of the starting assembly are insufficient to come to a conclusion on the influence of size on the disassembly. For example, in Fig. 6 two starting assemblies, $\mathrm{A}$ and $\mathrm{B}$, having the same size and mass are shown. But, in spite of their overall size and mass being comparable, the difference between the sizes of their quotients is considerable. Therefore, it can be seen that $\mathbf{M}_{\mathbf{S A}}$ alone is insufficient to draw an inference regarding the handling and the fixtures used. Therefore the average size/mass of the quotients is also considered. This value of this attribute also ranges from one to ten, where one indicates a very small average size/mass and ten a large size/mass. The rating reference is the same as that for the entire starting assembly.

\section{(c) Shape regularity of the starting assembly $\left(\mathbf{R}_{\mathrm{SA}}\right)$}

This attribute again influences the nature of handling and fixture requirements. The scale ranges 

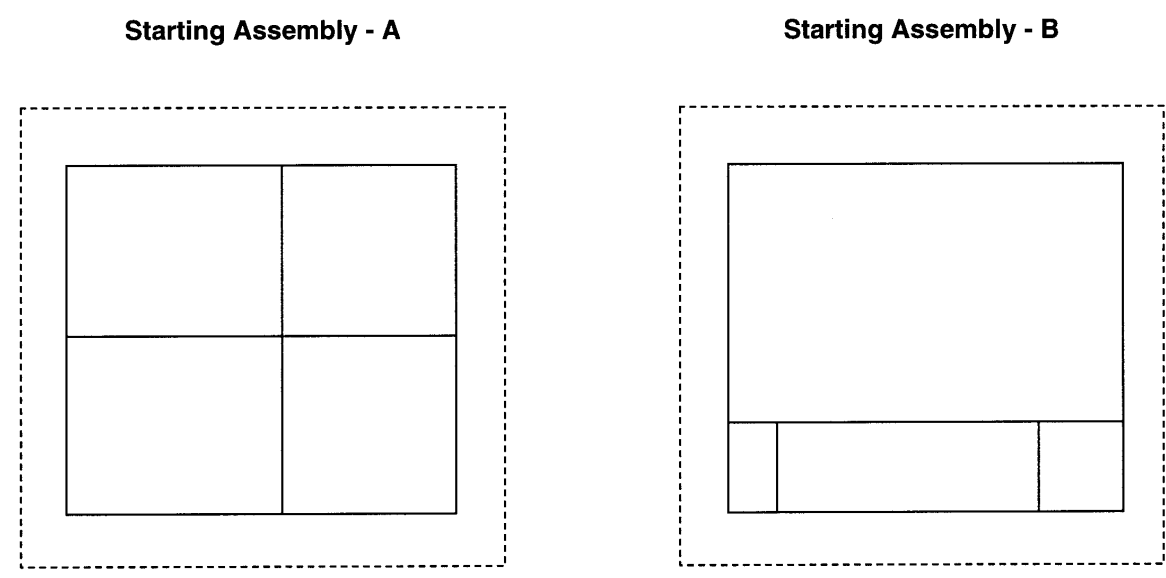

Fig. 6. Size/mass of starting assembly paradox.

from one for very regular shaped starting assemblies to ten indicating very irregular starting assemblies. The term "regular" is used in the geometric sense.

\section{(d) Average shape regularity of the quotients $\left(\mathbf{R}_{\mathbf{q}}\right)$}

The argument used to show the need for $\mathbf{M}_{\mathbf{S A}}$ and $\mathbf{M}_{\mathbf{q}}$ applies to this case as well. Therefore, the average shape regularity of the quotients is also considered.

\subsection{2. $\quad$ The resource requirements}

The primary DPGs formed are further subdivided into secondary DPGs based on the resource requirements for the disassembly. The resources refer to the tools, fixtures and other important equipment requirements needed to perform the disassembly. These attributes, however, deal only with what is required to perform the disassembly and not how to perform the disassembly. These attributes are:

(a) Average tool complexity.

(b) Average fixture complexity.

(c) Average operating and handling conditions.

(d) Auxiliary equipment.

(e) Relative disassembly duration.

\section{(a) Average tool complexity ( $\mathrm{T}$ )}

This refers to the tools used to perform the disassembly operations. The gradation of complexity is from simple standard tools to non-standard tooling. Now, there could be a number of tools used to undo a given fastener and those used in a particular disassembly facility would be chosen based on factors like economics, volume of disassembly carried out, safety, etc. that are specific to the facility. It also follows that these tools need not be the simplest tools with which the task could be performed. To keep the grouping independent of the specific capabilities of any particular disassembly facility, the "simplest" tool with which the given fastener can be undone with acceptable efficiency is taken as the basis for the rating of this attribute. For example, consider a dividend that requires the removal of a screw fastener. To accomplish this task it is found that a manual screwdriver or a pneumatically driven screwdriver could be employed. Technically, even a nuclear powered one could also be used. But as stated earlier, the simplest tool that can be used to perform this operation is considered determines the rating for this attribute which, in this case would be the manual screwdriver. This line of reasoning of using the "simplest" tool as the basis for the attribute rating is called as the Simplicity Concept.

The tooling requirement, for a product, includes those that may be required due to effects of usage on the dividends such as rusting, jamming of the quotients, and dirt accumulation.

\section{(b) Average fixture complexity (F)}

This attribute is a measure of the type of fixtures required to disassemble the product. The fixtures required are dependent on the operations that need to be performed on the dividend and the physical properties of the dividend such as its shape, size and mass. The fixture complexity varies from simple locators through to complex operation-specific fixtures. The simplicity concept is also used to reason about the fixtures. The fixtures considered must include those 
that may be required due to the effects of usage on the dividends.

\section{(c) Average operating and handling conditions $(\mathbf{H})$}

This is concerned with the specific handling and operating requirements for the disassembly of the dividend. For example, the disassembly of electronic products may require the use of a clean room or the disassembly of a product that results in the release of toxic gases may require a secluded area. Special handling may be required for hazardous quotients such as toxic quotients, radioactive quotients, and quotients having sharp edges. This attribute is a measure of the nature and extent of such requirements of the entire disassembly of the starting assembly and its R-moieties, by use of the simplicity concept and considerations of requirements due to usage effects on the dividends.

\section{(d) Auxiliary equipment and processes $\left(\mathrm{E}_{\mathrm{AD}}\right)$}

Though often not recognized as an important contributing factor to disassembly, auxiliary operations such as cleaning and inspection of the quotients and operations to facilitate ease and safety of handling are an integral part of disassembly. A specific instance of this last use of auxiliary operations to facilitate safety of handling is given by Hentschel et al. ${ }^{14}$ stating the case of the disassembly of CRTs. CRTs contain a vacuum, making them dangerous for handling and manipulation, so a specific operation to punch the CRT to release the vacuum is carried out. The gradation in the rating for a starting assembly varies from minimal requirements for auxiliary processes to complex processing requirements. All the ratings are made using the simplicity concept and the requirements due to usage effects on the dividends.

\section{(e) Disassembly duration $\left(\mathrm{N}_{\mathrm{D}}\right)$}

Time is an important resource and its use in disassembly is critical. The disassembly duration is the average time taken to disassemble the starting assembly down to all it's A- and F-moieties, making allowances for deviations due to expected usage effects on the dividends. It is assumed that the disassembly is accomplished using the equipment defined by the simplicity concept and that the disassembly of the intermediate dividends that are formed is done sequentially. A rating of one is given if the time required is of the order of four to five minutes and a rating of ten when it takes more than an hour to disassemble the product.

\subsection{Nature of similarity}

Due to the approximate nature of the values of attributes used for the DPG formation, an ordinal

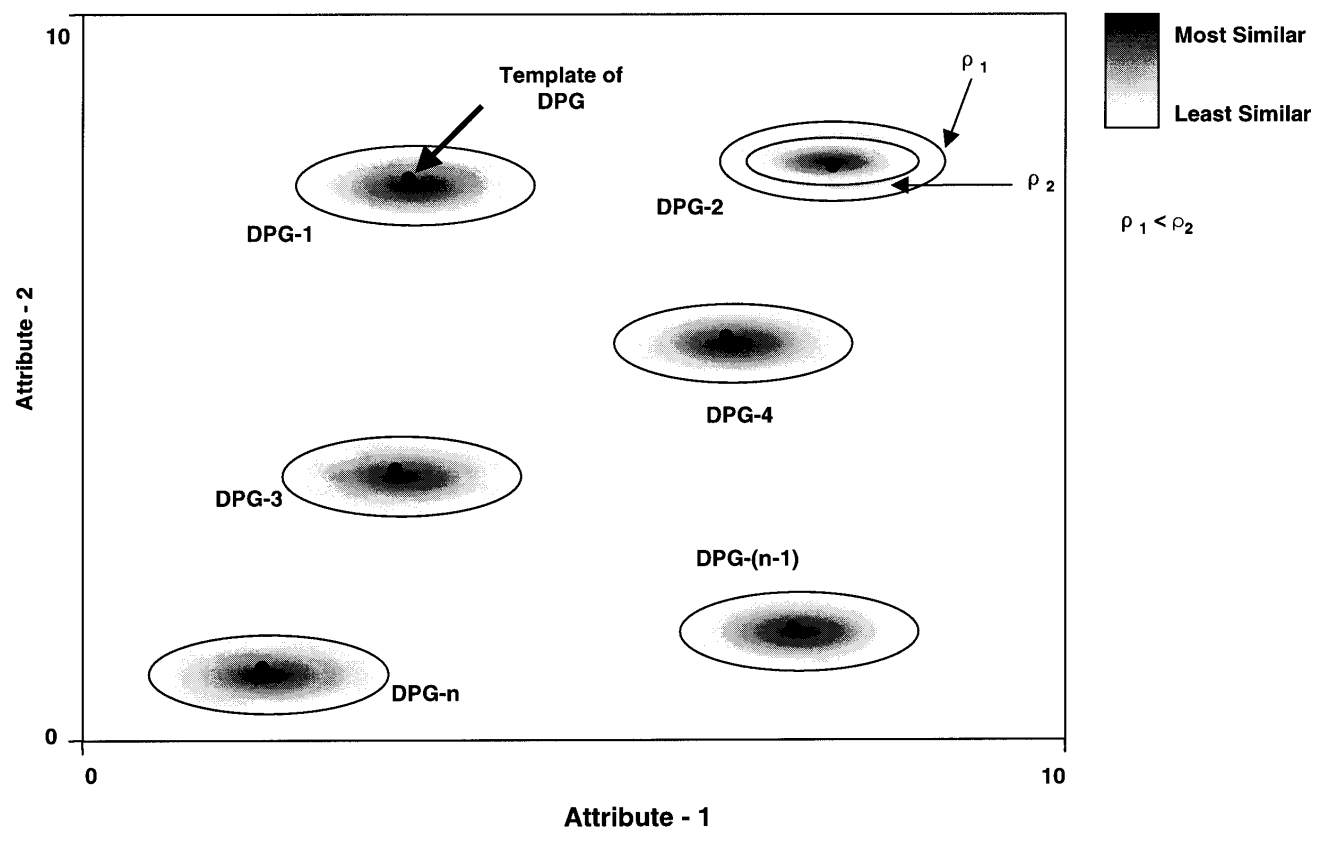

Fig. 7. DPG formation for a two-attribute case. 
approach to the attributes used rather than a cardinal approach. But a scale with values ranging from one to ten sometimes does not help to express the difference in similarity between products effectively. So the scale was made non-linear by using a basis function for each attribute that dictates the extent of similarity between two values of a given attribute. The basis function is assumed to be a Gaussian, with no reference to its statistical connotations. This function has a defining characteristic called the "flexibility coefficient" $(\sigma)$, which is set by the user and is a function of the attribute and the extent of non-linearity of the scale. The similarity threshold $(\rho)$ determines the extent of similarity required between the different starting assemblies during the DPG formation with a value of one indicating a complete match and lower values indicate lower degrees of similarity. The value of the similarity threshold $(\rho)$ is also set by the user depending on the requirement. Consider Fig. 7 for an illustration of the use of the basis function for a twoattribute case. It can be seen from Fig. 7 that the flexibility coefficient determines the rate of similarity variation and is indicated by the level of color change. For simplicity, in this paper, $\sigma$ is taken to be constant over the entire range of values of the attribute.

\section{3. "Growing Neural Gas" Network Model}

To recapitulate, the problem can be stated as, "The need to classify available data into representative clusters or subsets, where the properties of items in one cluster have similar properties but are different from those belonging to different clusters. (Information on the nature and number of clusters required being unavailable a priori.)"

With these constraints it was found suitable to use an adaptation of the "growing neural gas" 19,20 algorithm since it is an unsupervised, incremental neural network model, not requiring prior knowledge of the nature of the output or the size of the network. The "Growing Neural Gas" (GNG) network model $^{19,20}$ is an algorithm that performs 'topology learning'. It basically generates a graph structure that closely reflects the topology of the input data manifold. The main idea of the method is to successively add new units to an initially small network evaluating local statistical measures gathered during previous adaptation steps.

In a grouping application, as is the present case, it is the topology of the various inputs (the attributes of the product assemblies) that has to be obtained from which the groups are determined.

In the GNG algorithm the network is considered to consist of:

(a) A set of units or nodes, A, with each unit, $c \in A$, having an associated $n$-dimensional reference vector $w_{c}, n$ being the number of attributes. These units are the receptive fields of the different DPGs in the input space.

(b) A set of connections or edges, $C$, among the pairs of units. These serve to define the topological structure by connecting units having correlated activity.

The working of the algorithm can be explained easily for a two-dimensional case. Assume that the topology of the input space at some given time is as shown in the graph in Fig. 8(a). Each of the nodes represents the position of the units in the input space. An edge exists between nodes whose positions in the input space are correlated. When an input is added as shown in Fig. 8(b) all the units move towards it to an extent dependent on their distances from it. Depending on the distance of each of the units from the input, a local error variable is incremented and is represented by the stacks shown in Fig. 8(c). A variable called the 'age' is attached to each of the edges to determine the nature of the adaptation that takes place. It is a measure of the correlation in movements between connected units. So an increase in age indicates a decrease in correlation between the connected units. A maximum age limit is set depending on the requirements and when an edge reaches this age it is removed. As inputs are presented to the network, the units move to adapt to the new inputs resulting in a distortion error. So in the GNG algorithm after predetermined intervals a new unit is inserted between the unit having the highest error and the topological neighbor that has the highest error. So as new inputs are presented the earlier learnt topology is retained. This insertion rate $(\lambda)$ is constant and is preset before running the network

The advantages of the GNG algorithm that are also of specific relevance in this application are:

(a) The number of clusters that need to be formed does not require to be specified initially. This is an important advantage as the products considered are totally different and there is no way of determining the number of DPGs a priori. 
(a)
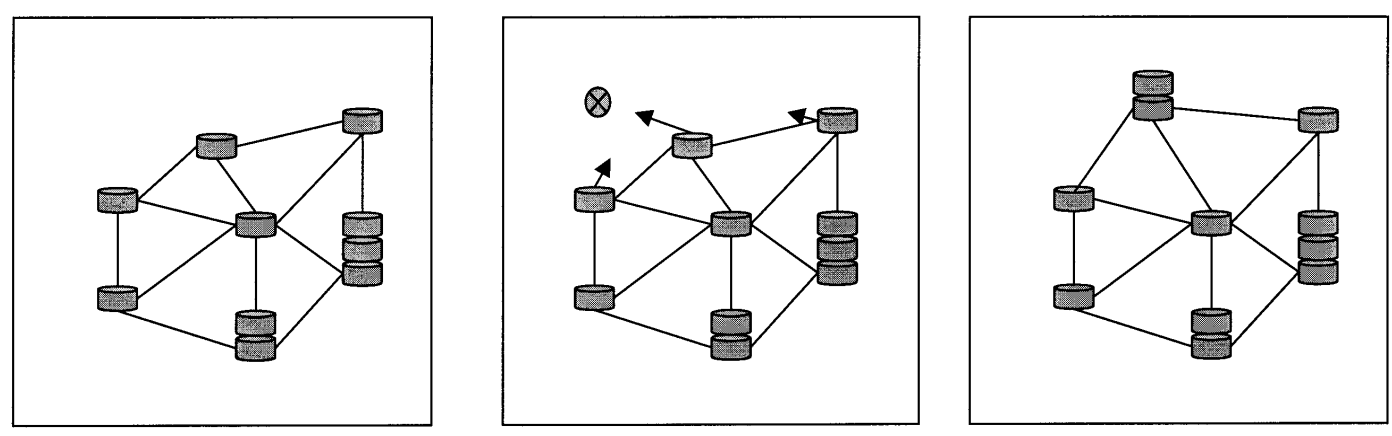

Error Variable

Q Presented Input

Fig. 8. Basic adaptation principle of the growing neural gas algorithm. ${ }^{16}$

(b) All the parameters are constant and also the number of adaptation steps need not be specified beforehand.

\section{1. $\quad$ The modified growing neural gas algorithm}

We have modified the original GNG algorithm to suit the present disassembly application. This modified GNG algorithm is described in a stepwise fashion as follows:

1. Start with two units $a$ and $b$, chosen randomly from the input data, defined by the reference vectors, $w_{a}$ and $w_{b}$. Initialize the connection set to contain an edge between $a$ and $b$ and set the age of this edge to zero. Therefore, $\operatorname{age}_{(a, b)}=0$. The age of an edge serves as an indication for removing edges, generated earlier, which have become obsolete due to the motions of the connected units.

2. The disassembly attributes are presented as a vector, $\mathbf{p}$, to the network.

3. Determine units $s_{1}$ and $s_{2}\left(s_{1}, s_{2} \in A\right)$ such that:

$$
\begin{aligned}
& \sum_{i=1}^{n} W_{i} \exp \left[-\frac{\left(p_{i}-w_{s_{1} i}\right)^{2}}{2 \sigma_{i}^{2}}\right] \\
& \geq \sum_{i=1}^{n} W_{i} \exp \left[-\frac{\left(p_{i}-w_{c i}\right)^{2}}{2 \sigma_{i}^{2}}\right] \quad(\forall c \in A) \\
& \quad\left(\forall c \in A \backslash s_{1}\right)
\end{aligned}
$$

$$
\begin{aligned}
\sum_{i=1}^{n} W_{i} \exp \left[-\frac{\left(p_{i}-w_{s_{2} i}\right)^{2}}{2 \sigma_{i}^{2}}\right] \\
\geq \sum_{i=1}^{n} W_{i} \exp \left[-\frac{\left(p_{i}-w_{c i}\right)^{2}}{2 \sigma_{i}^{2}}\right]
\end{aligned}
$$

$$
\sum_{i=1}^{n} W_{i}=1
$$

where

$$
\begin{aligned}
& W_{i}= \text { Relative importance of the } i \text { th } \\
& \text { attribute }
\end{aligned}
$$

This deviation from the conventional "growing neural gas" (originally proposed by Fritzke ${ }^{20}$ ) is due to the assumption about the nature of "similarity" mentioned earlier. The relative importance weights, $W$, for each of the attributes indicates the relative priorities of the different attributes during the DPG formation, determined by the application specific requirement. 
4. Insert a connection between $s_{1}$ and $s_{2}$, if it does not already exist. Either way 'refresh' the connection between $s_{1}$ and $s_{2}$ by setting the age to zero.

$$
\operatorname{age}_{\left(s_{1}, s_{2}\right)}=0
$$

5. Add the squared distance between the input vector and the nearest unit $\left(s_{1}\right)$ in the node space to a local error variable. The norm is Euclidean.

$$
\Delta E_{s_{1}}=\left\|w_{s_{1}}-p\right\|^{2}
$$

6. Move $s_{1}$ and its direct topological neighbors (the units connected by edges to $s_{2}$ ) by fractions $\varepsilon_{b}$ and $\varepsilon_{n}$, respectively, of the total distance:

$$
\begin{aligned}
\Delta w_{i} & =\varepsilon_{n}\left(p-w_{i}\right) \quad\left(\forall i \in N_{s_{1}}\right) \\
\Delta w_{s_{1}} & =\varepsilon_{b}\left(p-w_{s_{1}}\right)
\end{aligned}
$$

7. Increment the ages of all the edges emanating from $s_{1}$ :

$$
\operatorname{age}_{\left(s_{1}, j\right)}=\operatorname{age}_{\left(s_{1}, j\right)}+1 \quad\left(\forall i \in N_{s_{1}}\right)
$$

8. Remove edges that have an age greater than $a_{\max }$. If this results in units with no emanating edges then remove them as well.

9. If the number of input signals generated so far is an integer multiple of a parameter $\lambda$, then insert a new unit using the following procedure:

(a) Determine the unit $q$ having the maximum accumulated error

$$
E_{q} \geq E_{c} \quad(\forall c \in A)
$$

(b) Insert a new unit $r$ halfway between $q$ and one of its neighbors $f$ having the highest accumulated error

$$
\begin{aligned}
A & =A \cup\{r\} \\
w_{r} & =0.5\left(w_{q}+w_{f}\right)
\end{aligned}
$$

(c) Insert edges connecting the new unit $r$ with the units $q$ and $f$ and remove the original edge between $q$ and $f$

$$
C=C \cup\{(r, q),(r, f)\}(C=C \backslash(q, f))
$$

(d) Decrease the error variables of $q$ and $f$ by multiplying them with a constant $\alpha$

$$
\begin{aligned}
& \Delta E_{q}=-\alpha E_{q} \quad(0<\alpha<1) \\
& \Delta E_{f}=-\alpha E_{f}
\end{aligned}
$$

(e) Interpolate the error variable of $r$ from $q$ and $f$

$$
E_{r}=0.5\left(E_{q}+E_{f}\right)
$$

6. Decrease all the error variables by multiplying them with a constant factor, $\beta$

$$
\Delta E_{c}=-\beta E_{c} \quad(\forall c \in A),(0<\beta<1)
$$

7. If all the inputs are classified with the minimum similarity threshold then stop, otherwise continue with step 2 . This similarity threshold, $\rho$, is preset and has value between 0 and $1(0 \leq \rho \leq 1)$, where 1 indicates complete similarity and lower values indicate a lower similarity. When each of the members of the input set are presented to the network, the classification is complete, if:

$$
\left(\sum_{i=1}^{n} W_{i} \exp \left[-\frac{\left(p_{i}-w_{j i}\right)^{2}}{2 \sigma_{i}^{2}}\right]-\rho\right) \geq 0
$$

For further specific details of the "growing neural gas" algorithm the reader is referred to Fritzke ${ }^{19}$ and the handbook of neural computation. $^{20}$

\subsection{Implementation}

The DPG formation using the GNG algorithm was implemented using the MATLAB software (Ver 5.1.0.42) on a HP C-200 workstation. The starting assemblies as listed in Table 1 were used for the DPG formation. Diverse starting assemblies were chosen to illustrate the utility of the methodology.

Due to the absence of a disassembly specific database of disassembly times, sequences and resources used, the values of the attributes for the different starting assemblies were derived based on several references ${ }^{21-27}$ and expert opinion. The primary DPGs obtained for the assemblies listed in Table 1, for different values of the similarity threshold $(\rho)$ are shown in Table 2. As expected, the DPGs become smaller as the value of $\rho$ is increased.

Two of the primary DPGs formed at an arbitrarily chosen value of $\rho=0.5$ were chosen to demonstrate the formation of the secondary DPGs. The secondary DPGs formed for different similarity 
Table 1. Products to be grouped.

\begin{tabular}{|l|c|c|c|c|c|c|c|c|c|}
\hline \multirow{2}{*}{ Product } & \multicolumn{4}{|c|}{ Product Structure } & \multicolumn{5}{|c|}{ Resource Requirements } \\
\cline { 2 - 9 } & $\mathbf{M}_{\mathbf{S A}}$ & $\mathbf{M}_{\mathbf{q}}$ & $\mathbf{R}_{\mathbf{S A}}$ & $\mathbf{R}_{\mathbf{q}}$ & $\mathbf{T}$ & $\mathbf{F}$ & $\mathbf{H}$ & $\mathbf{E}_{\mathbf{A D}}$ & $\mathbf{N}_{\mathbf{D}}$ \\
\hline 1. Gas Pressure Regulator & 1.5 & 1 & 2 & 2.5 & 5.0 & 2 & 0 & 1 & 2 \\
2. Air Pressure Regulator & 1.5 & 0.8 & 2 & 3 & 2 & 2 & 0 & 1 & 3 \\
3. Table Fan & 3.5 & 3 & 3 & 3 & 2 & 1 & 0 & 1 & 3 \\
4. Toaster-Oven & 4.6 & 2.8 & 2 & 2.5 & 2 & 1 & 0 & 2 & 3.5 \\
5. Refrigerator & 8 & 6 & 4 & 5 & 7 & 5 & 5 & 7 & 8 \\
6. Car Engine & 8 & 4 & 8 & 7 & 8 & 8 & 4 & 4 & 10 \\
7. Automobile Carburetor & 2.5 & 1.3 & 7 & 7 & 4 & 1 & 0 & 4 & 5 \\
8. Vacuum Cleaner & 5 & 2.7 & 6 & 4 & 2 & 1 & 0 & 2 & 4 \\
9. Disposable Camera & 2.5 & 1.0 & 4 & 5 & 2 & 2 & 6 & 1 & 1.5 \\
10. Coffee Maker & 3.3 & 2.5 & 3 & 3 & 2 & 1 & 0 & 2 & 4 \\
11. HP-Printer & 4.5 & 2.7 & 3 & 4 & 3 & 2.5 & 0 & 0.5 & 4.5 \\
\hline
\end{tabular}

Table 2. Primary DPGs formed.

\begin{tabular}{|c|c|}
\hline $\begin{array}{c}\text { Similarity } \\
\text { Threshold } \\
(\rho)\end{array}$ & $\begin{array}{c}\text { Primary DPGs } \\
\left(\varepsilon_{b}=0.4, \varepsilon_{n}=0.01, \lambda=6, \alpha=0.5, \beta=0.002, \sigma_{1}=0.8, \sigma_{2}=0.8, \sigma_{3}=1, \sigma_{4}=1\right)\end{array}$ \\
\hline 0.1 & $\{1,2,3,4,5,7,8,9,10,11\},\{3,4,5,6,8,9,10,11\},\{1,2,3,4,5,8,9,10,11\}$ \\
0.2 & $\{1,2,3,4,7,9,10,11\},\{5,6,8,9,11\},\{1,2,3,4,8,10,11\},\{1,2,3,4,5,7,8,9,10,11\}$ \\
0.3 & $\{1,2,3,7,9,10,11\},\{5,6\},\{1,2,3,4,8,10,11\},\{2,3,4,8,9,10,11\},\{3,4,8,11\},\{1,2,3,7,9,10,11\}$ \\
0.4 & $\{1,2\},\{5\},\{6\},\{10\},\{7,9\},\{3,9,10,11\},\{8,11\},\{3,4,8,10,11\},\{3,9,10,11\},\{4,8,11\}$ \\
$\mathbf{0 . 5}$ & $\{\mathbf{1}, \mathbf{2}\},\{6\},\{3,4,10,11\},\{3,10,11\},\{8,11\},\{5\},\{7,9\},\{9\},\{\mathbf{3}, \mathbf{4}, \mathbf{8}, \mathbf{1 0}, \mathbf{1 1}\}$ \\
0.6 & $\{1,2\},\{6\},\{3,10\},\{8\},\{5\},\{7\},\{9\},\{11\},\{4,11\},\{9\}$ \\
0.7 & $\{1\},\{2\},\{3\},\{4\},\{5\},\{6\},\{7\},\{8\},\{9\},\{10\},\{11\}$ \\
0.8 & $\{1\},\{2\},\{3\},\{4\},\{5\},\{6\},\{7\},\{8\},\{9\},\{10\},\{11\}$ \\
0.9 & $\{1\},\{2\},\{3\},\{4\},\{5\},\{6\},\{7\},\{8\},\{9\},\{10\},\{11\}$ \\
\hline
\end{tabular}

Table 3. Secondary DPGs. DPG $\mathrm{P} 1=\{3,4,8,10,11\}, \mathrm{DPG}_{\mathrm{P} 2}=\{1,2\}$.

\begin{tabular}{|c|c|c|}
\hline \multirow{2}{*}{$\begin{array}{c}\text { Similarity } \\
\text { Threshold }\end{array}$} & \multicolumn{2}{|c|}{ Secondary DPGs Formed } \\
\cline { 2 - 3 }$(\rho)$ & From $\left.\varepsilon_{b}=0.4, \varepsilon_{n}=0.01, \lambda=6, \alpha=0.5, \beta=0.002, \sigma_{1}=\sigma_{2}=\sigma_{3}=\sigma_{4}=\sigma_{5}=1\right)$ \\
\hline 0.1 & $\{3,4,8,8,10,11\}$ & From $\mathrm{DPG}_{\mathrm{P} 2}=\{1,2\}$ \\
0.2 & $\{3,4,8,10,11\}$ & $\{1,2\}$ \\
0.3 & $\{3,4,8,10,11\}$ & $\{1,2\}$ \\
0.4 & $\{3,4,8,10,11\}$ & $\{1,2\}$ \\
0.5 & $\{3,4,8,10,11\}$ & $\{1,2\}$ \\
0.6 & $\{3,4,8,10,11\}$ & $\{1,2\}$ \\
0.7 & $\{3,4,8,10,11\},\{3,4,8,10\}$ & $\{1,2\}$ \\
0.8 & $\{3,8,10,11\},\{3,4,8,10\}$ & $\{1\},\{2\}$ \\
0.9 & $\{11\},\{4,8,10\},\{3,4\},\{3\}$ & $\{1\},\{2\}$ \\
1.0 & $\{3\},\{4\},\{11\},\{8,10\}$ & $\{1\},\{2\}$ \\
\hline
\end{tabular}

threshold values is as shown in Table 3 . The primary DPGs chosen for this purpose is shown in bold lettering in Table 2. As in the formation of primary DPGs, the secondary DPGs become smaller as the value of $\rho$ is increased.
The values of the other parameters used in this implementation are provided in Table 4.

\subsubsection{Comments on results}

An important factor to be noted in the above 
Table 4. Network parameter values.

\begin{tabular}{|c|c|c|}
\hline \multirow{2}{*}{} & \multicolumn{2}{|c|}{ Network Parameters } \\
\cline { 2 - 3 } & Primary DPG & Secondary DPG \\
\hline$\varepsilon_{b}$ & 0.4 & 0.4 \\
$\varepsilon_{n}$ & 0.01 & 0.01 \\
$\lambda$ & 6 & 6 \\
age $_{\max }$ & 7 & 7 \\
$\alpha$ & 0.5 & 0.5 \\
$\beta$ & 0.002 & 0.002 \\
$\sigma_{1}$ & 0.8 & 1 \\
$\sigma_{2}$ & 0.8 & 1 \\
$\sigma_{3}$ & 1 & 1 \\
$\sigma_{4}$ & 1 & 1 \\
\hline
\end{tabular}

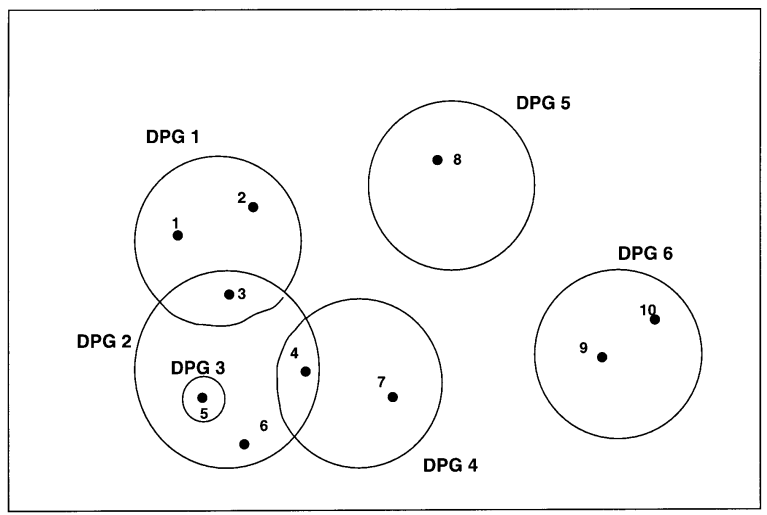

- = Starting Assembly

Fig. 9. Venn diagram of DPGs formed.

implementation is that a compounded similarity value is not obtained, i.e., the similarity of the starting assemblies in a particular secondary DPG is independent of their similarity values in the primary DPG. Also it can be seen that many of the primary DPGs obtained have common constituents. This can be explained using Fig. 9. The algorithm performs the clustering till all the starting assemblies are classified. Rather than trying to determine the best DPG form based on criteria like - group having maximum members, group with highest average similarity and so on, it is desirable to consider all the obtained groups as they reflect the correlation between the different starting assemblies.

\subsection{Network parameters}

The parameter, $\lambda$, is the rate at which new nodes are to inserted into the network. This does away with the need to perform an adaptation at every step. If adaptation is not required to move units over large distances, the value of $\lambda$ is kept small.

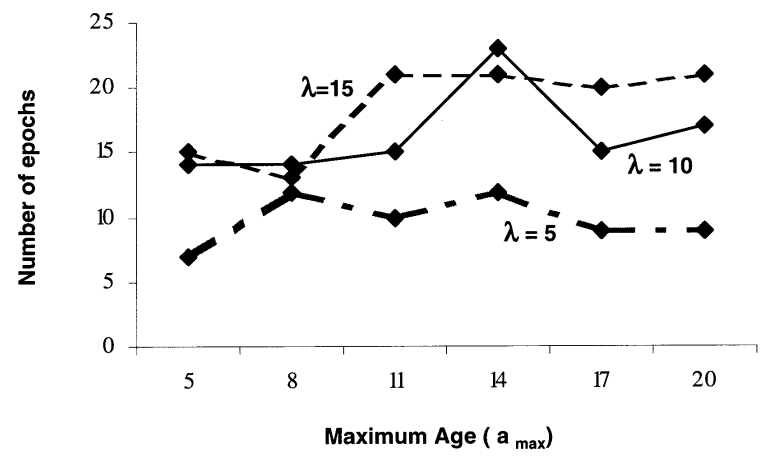

(a)

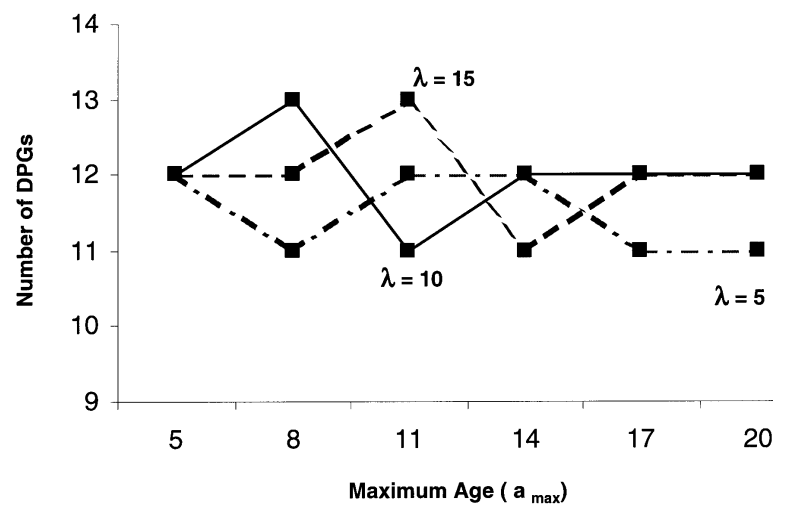

(b)

Fig. 10. (a) Variation of number of epochs with the maximum age $\left(a_{\max }\right)$. (b) Variation of number of DPGs formed with the maximum age $\left(a_{\max }\right)$.

The "age" of an edge is indicative of the "distance" and decrease in correlation of the connected nodes thereof. So by setting the maximum age $\left(a_{\max }\right)$, the limit of correlated activity between connected nodes is set. The relationship between the number of adaptive epochs, $\lambda$ and $a_{\max }$ for a set of 15 inputs is shown in Figs. 10(a) and 10(b). Similar investigations were carried out for different numbers of inputs and it was seen that the number of groups formed is relatively insensitive to these parameters within certain range of values.

The values of $\alpha$ and $\beta$ were determined by trial and error and in this application, $\beta \ll \alpha$, was found to work well. The values of $\varepsilon_{n}$ and $\varepsilon_{b}$ were also determined by trial and error. For the implementation at $\rho=0.5$ described above, the algorithm took $1.30 \mathrm{CPU}$ seconds to form the primary DPGs. The time taken by the GNG algorithm to form the DPGs, in general, increases as $\rho$ increases. This time also increases with the size of the disassembly attribute vectors. 


\section{Discussion}

Analyzing the results in Table 3 , it is seen that the grouping based on the attributes chosen produced results that can be intuitively validated. For example, the air-pressure regulator and the gas-pressure regulator display a high similarity at both levels because the two products have large structural and resource requirement similarity.

The other primary DPG of interest is \{Fan, Toaster-oven, Vacuum cleaner, Coffeemaker, Printer $\}$. It is apparent that these products are similar from the first tier attributes dealing with the product structure. From the secondary DPGs formed it can be seen that this group has a high similarity based on resource requirements. It is implicit in this conclusion that similarity does not imply congruence. This means that the fact that if two products both have the same ranking for a particular attribute such as tool complexity, does not mean that they use the same tools. Therefore, it may not be possible to disassemble all the members of a given DPG with the same resources, but these members certainly have a high potential to use common disassembly resources.

Though the attributes do not directly address the cost issues involved, they are indirectly addressed. This argument is based on the assumption that standard equipment is cheaper than non-standard equipment. Though a number of anomalies exist to prevent the acceptance of this argument, it is largely true.

The model presented in this study is a simplified version of the actual problem and there remain a number of issues to be addressed in greater depth such as the exact nature of the basis function for each attribute and the calculation of the flexibility coefficient $(\sigma)$. These would require empirical investigations. Also the range of the similarity threshold $(\rho)$ over which the DPGs formed are industrially relevant are questions that require further investigation. Here, we have used only limited number of attributes so as to obtain an initial rough grouping. Further investigation is required to identify factors that have to be considered to obtain finer subdivisions of each of the groups already formed.

Other possible applications of this methodology could be in the development of generic Design for Disassembly (DFD) guidelines for diverse products. Additionally, DPGs may help in determining the scope or extent of variability during the development of a variant design from the disassembly perspective. This aspect of the extent of variability for the variant designs is especially important with the increas- ing trend towards mass customization of consumer products. In the light of increased research in disassembly related issues, establishing a database of disassembly information for diverse products could be of immense utility to the research and industrial community.

\section{Conclusion}

A framework for the formation of product groups from the disassembly perspective as a starting aggregation towards the formation of flexible disassembly facilities and cells has been proposed. A neural network model based on the "growing neural gas algorithm" was used to demonstrate the implementation of the proposed framework proposed using eleven test products. Though the concept of grouping is not novel in itself, earlier applications have been limited in scope and differed in the intended area of application. The proposed framework is generic to form groups of products from a diverse product universe that have common disassembly characteristics. The framework presented is however a simplified one and much research work needs to be done to bring to a level so as to be deployable in industry.

\section{Acknowledgments}

This work has been supported in part by the US National Science Foundation Grant \#BES 9727136.

\section{References}

1. G. A. Keoleian and D. Menerey, "Sustainable development by design: Review of life-cycle design and related appoaches", Air \&G Waste 44 (1994), 645-668.

2. N. M. Darnall, I. G. Nehman, J. W. Priest and J. Sarkis, "A review of environmentally conscious manufacturing theory and practices", International Journal of Environmentally Conscious Design 86 Manufacturing 3(2) (1994), 49-57.

3. H. C. Zhang, T. C. Kuo, H. Lu and S. H. Huang, "Environmentally conscious design and manufacturing: A state-of-the-art survey", Journal of Manufacturing Systems 16(5) (1997), 352-371.

4. L. K. Moyer and S. M. Gupta, "Environmental concerns and recycling/disassembly efforts in the electronics industry", Journal of Electronics Manufacturing 7(1) (1997), 1-22.

5. A. Gungor and S. M. Gupta, "Issues in environmentally conscious manufacturing and product recovery: A survey", Computers and Industrial Engineering 36(4) (1999), 811-853.

6. F. Jovane, L. Alting, A. Armillota, W. Eversheim, K. Feldmann, G. Seliger and N. Roth, "A key issue in product life-cycle: Disassembly", Annals of the CIRP 42(2) (1993), 651-658. 
7. J. Sarkis, "Supply chain management and environmentally conscious design and manufacturing", International Journal of Environmentally Conscious Design and Manufacturing 4(2) (1995), 43-52.

8. R. Uzsoy, "Production planning for companies with product recovery and remanufacturing capability", Proceedings of the IEEE International Symposium on Electronics and the Environment, San Francisco, 1997, pp. 285-289.

9. T. Hoshino, K. Yura and K. Hitomi, "Optimization analysis for recycle-oriented manufacturing systems", International Journal of Production Research 33(8) (1995), 2069-2078.

10. J. A. Stuart, J. C. Ammons and L. J. Turbini, "A product and process selection model with multidisciplinary environmental considerations", to appear in Operations Research.

11. S. M. Gupta and K. N. Taleb, "Scheduling disassembly", International Journal of Production Research 32(8) (1994), 1857-1866.

12. A. Kochan, "In search of a disassembly factory", Assembly Automation 15(4) (1995), 16-17.

13. S. M. Gupta and C. R. McLean, "Disassembly of products", Computers in Industrial Engineering 31(1\&2) (1996), 225-228.

14. C. Hentschel, G. Seliger and E. Zussman, "Grouping of used products for cellular recycling systems", Annals of the CIRP 44(1) (1995), 11-14.

15. "Development of flexible tools specially designed for disassembly", Institute for Manufacturing $\mathrm{Au}-$ tomation and Production Systems, University of Erlangen-Nuremberg, http://www.faps.uni-erlangen.de/presentation/ recycling/HTML/bohr1eng.html

16. M. P. Groover, Automation, Production Systems and Computer Integrated Manufacturing, 2nd edition, Prentice Hall, 1989.
17. T. C. Chang, Expert Systems in Process Planning, Addison-Wesley, 1990.

18. P. Veerakamolmal and S. M. Gupta, "Design of an integrated component recovery system", Proceedings of the IEEE International Symposium on Electronics and the Environment, Oak Brook, Illinois, 1998, pp. 285-289.

19. Fritzke, B., "A growing neural gas network learns topologies", Advances in Neural Information Processing Systems 7, MIT Press, Cambridge MA, 1995, pp. 625-632.

20. E. Fiesler and R. Beale, Eds., "Unsupervised ontogenic networks", Handbook of Neural Computation, Institute of Physics Publishing Ltd. and Oxford University Press Inc., 1997, pp. C2.4:1-C2.4:16.

21. C. M. Rose, K. Ishii and K. Masui, "How product characteristics determine end-of-life strategies", Proceedings of the IEEE International Symposium on Electronics and the Environment, Oak Brook, Illinois, 1998, pp. 322-327.

22. K. D. Penev and A. J. deRon, "Development of disassembly line for refrigerators", Industrial Engineering 26(11) (1994), 50-53.

23. R. Callahan, Final Term Report for EMGT 354, Engineering Management Department, University of Missouri-Rolla, Winter Semester, 1998.

24. I. Cackler, Final Term Report for EMGT 354, Engineering Management Department, University of Missouri-Rolla, Winter Semester, 1998.

25. E. Ildeniz, Final Term Report for EMGT 354, Engineering Management Department, University of Missouri-Rolla, Winter Semester, 1998.

26. S. Viswanathan, Final Term Report for EMGT 354, Engineering Management Department, University of Missouri-Rolla, Winter Semester, 1998.

27. Springfield Remanufacturing Center (SRC), Springfield, Missouri, 1998. 
Copyright $\odot 2003$ EBSCO Publishing 